\title{
Brote de tiña por Trichophyton tonsurans en una escuela básica de Valparaíso, Chile
}

\author{
Rodrigo Cruz Ch. ${ }^{1}$, Constanza Yáñez H. ${ }^{2}$, Laura Carvajal S. ${ }^{3}$, Peggy Vieille ${ }^{3}$ y Carolina Barrientos ${ }^{2}$
}

\section{Ringworm of Trichophyton tonsurans outbreak in a basic school in Valparaíso, Chile}

Background: Ringworm are skin and its annexes infections. Trichophyton tonsurans is an emergent anthropophilic fungus that might cause outbreaks. Aim: To describe a ringworm outbreak by T. tonsurans in an elementary school in Valparaíso, Chile. Methods: A descriptive study was run between June and August, 2018. Students and his closest relatives in contact with them who were clinically diagnosed with ringworm have been considered a case. Mycological studies of samples had been carried out through morphophysiology tests. Data are shown in numbers and percentages. Results: There were 18 cases, 16 kids (15 from kindergarten) and 2 adults. Attack rate was $68 \%$ in kindergarten. Nine cases were boys with average age of 6 years old. For 14 cases their nationality was Chilean and in 1 case Haitian. First case was a Chilean boy, and primary case was a Haitian boy. The most frequent location was face. Ten cases yielded positive cultures for T. tonsurans. Most of the patients needed terbinafine treatment after a therapeutic failure performed with clotrimazole. Conclusion: T. tonsurans outbreak ocurred mainly in kindergarten male. The most frequent location was face. Most of the patients needed terbinafine treatment after a therapeutic failure with clotrimazole.

Keywords: School outbreak; ringworm; Trichophyton tonsurans.

Palabras clave: Brote escolar; tiña; Trichophyton tonsurans.

\section{Introducción}

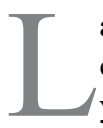
as tiñas son infecciones fúngicas que afectan estructuras queratinizadas como la piel, las uñas y los pelos ${ }^{1,2}$. Son provocadas por dermatofitos, uno de los grupos más antiguos de microorganismos que han sido reconocidos como agentes de enfermedades en humanos. Actualmente, se describen nueve géneros y más de 50 especies en este grupo: 16 Trichophyton, 1 Epidermophyton, 9 Nannizzia, 3 Microsporum, 1 Lophophyton, 21 Arthroderma, 1 Ctenomyces, 3 Paraphyton y 1 Guarromyces gen.nov ${ }^{3}$. Cada especie tiene un solo nombre válido y para las especies "borderline" aún son necesarios estudios más detallados para establecer su mejor clasificación ${ }^{3}$. Si bien el número de géneros aumentó desde la tradicional clasificación de Emmons de 1934 (Trichophyton, Microsporum y Epidermophyton), las especies que son de importancia para el laboratorio clínico ahora pertenecen a grupos más pequeños, lo que facilita su identificación ${ }^{3}$.

Dentro del género Trichophyton, T. rubrum es el más frecuente en la mayoría de los aislados en tiña de cuerpo y onicomicosis en las distintas regiones y países ${ }^{4,5}$. Trichophyton tonsurans es un hongo antropofílico emergente que se trasmite de persona a persona por contacto directo o indirecto a través de fómites ${ }^{6}$. Inicialmente se describió como una especie propia de los países de la cuenca mediterránea, tales como España, Portugal, sur de Francia, Italia y Grecia; sin embargo, hoy se considera que tiene una distribución mundial, siendo frecuente en E.U.A., Canadá y México como agente de tiña capitis ${ }^{6,7}$. En Centroamérica y América del Sur se han descrito aislamientos frecuentes en los últimos años, especialmente en niños ${ }^{6,7}$. En Chile, desde el año 2012 se ha demostrado la circulación de este agente en tiña capitis, probablemente influenciado por el aumento de la inmigración en los últimos años ${ }^{8}$.

Las características de las colonias de esta especie son bastante variables; su aspecto es aterciopelado o mullida, surcada radial o irregularmente, de color blanco a grisácea, a veces con centro oliváceo o rosado; el reverso puede ser de color caoba-rojo, amarillo o marrón y, a veces puede difundir un pigmento rojizo oscuro al medio 9 . En la microscopía se observan abundantes microconidios de tamaño variable, sésiles, clavados, a veces globosos. Los macroconidios, cuando están presentes, son variables, a menudo algo gruesos, de 2-6 células, cilíndricos o con forma de cigarro, de 10-65 $\times$ 4-12 $\mu \mathrm{m}$. Las clamidosporas terminales e intercaladas son abundantes ${ }^{9,10}$

El objetivo del presente trabajo fue describir un brote de tiña por T. tonsurans en una escuela básica de Placilla, Valparaíso, además de revisar las características clínicas, microbiológicas y epidemiológicas de esta infección.
'Laboratorio de Micología Universidad de Valparaíso. Infectología Hospital Carlos van Buren de Valparaíso. Chile. ${ }^{2}$ Centro de Salud familiar de Placilla, Valparaíso. Chile. ${ }^{3}$ Laboratorio de Micología Universidad de Valparaíso.Chile.

El autor declara no tener conflictos de interés.

Financiamiento: Universidad de Valparaíso.

Recibido: 4 de diciembre de 2018 Aceptado: 1 de abril de 2019

Correspondencia a: Rodrigo Cruz Choappa. rodrigo.cruz@uv.cl 


\section{Materiales y Método}

Estudio descriptivo de un brote escolar de tiña por $T$. tonsurans ocurrido entre junio y agosto del año 2018.

El 18 de junio de 2018, el Centro de Salud Familiar de Placilla notificó al servicio regional de salud la presencia de siete casos de tiña refractarias a tratamiento con clotrimazol en crema al $1 \%$ en alumnos de la Escuela Básica México de Placilla en Valparaíso. Debido a esto, en el mes de julio se contactó al laboratorio de Micología de la Universidad de Valparaíso para toma de muestra a los niños y padres afectados en el mismo colegío. Se entrevistó a los padres o a algún familiar directo y mediante una encuesta se recogió información sobre sexo, edad, nacionalidad, características clínicas y tratamiento de la infección. Cada familiar firmó un consentimiento informado autorizando la reproducción de imágenes de las lesiones de los niños y la publicación de los antecedentes demográficos. También se realizó un seguimiento en el centro de salud familiar para determinar si hubo respuesta clínica al tratamiento indicado.

\section{Definición de caso}

Se consideró como caso a todo niño de la Escuela Básica México y a familiares directos que estuvieron en contacto con ellos, que entre junio y agosto del 2018 tuvieron el diagnóstico clínico de tiña (lesiones típicas en cualquier localización del cuerpo), con o sin confirmación micológica (Figuras 1-3).

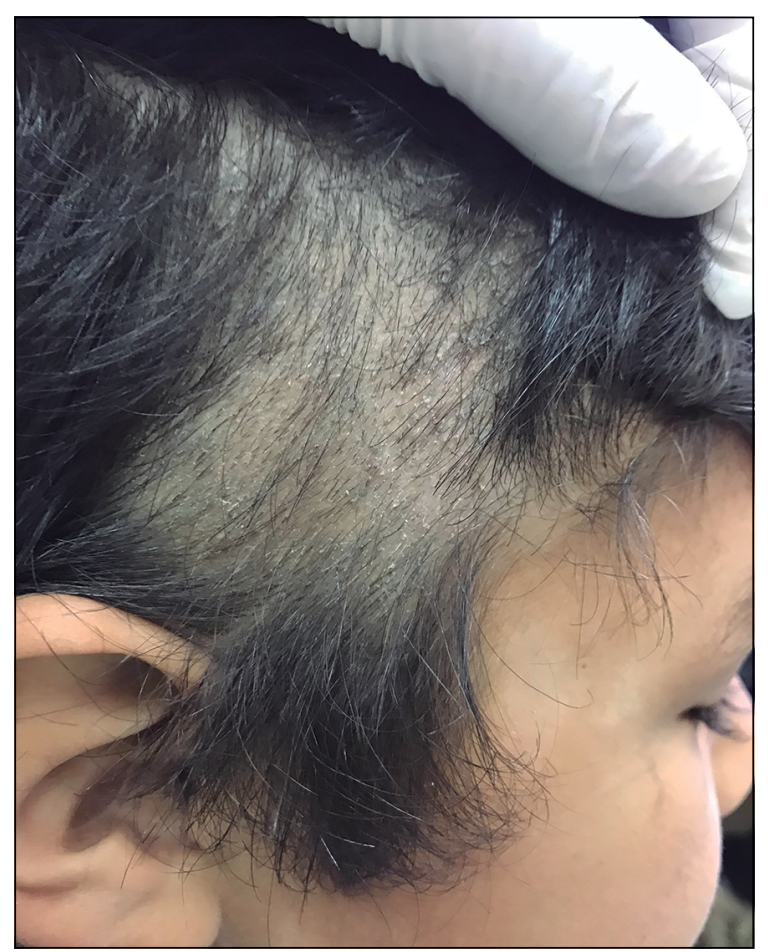

\section{Toma de muestra e identificación de especie}

Las muestras de las lesiones fueron obtenidas por personal entrenado y depositadas en placas de Petri estériles para evitar su contaminación. El cultivo se realizó en agar Sabouraud glucosado y agar Lactrimel, ambos adicionados con cloranfenicol $(125 \mu \mathrm{g} / \mathrm{ml})$ e incubadas a 27 y $37^{\circ} \mathrm{C}$ durante dos semanas. La identificación de la especie fue en base a su morfo-fisiología (Figuras 4 y 5 ).

\section{Análisis de resultados}

Los datos se recogieron en una planilla Excel y los resultados se expresaron en números y porcentajes.

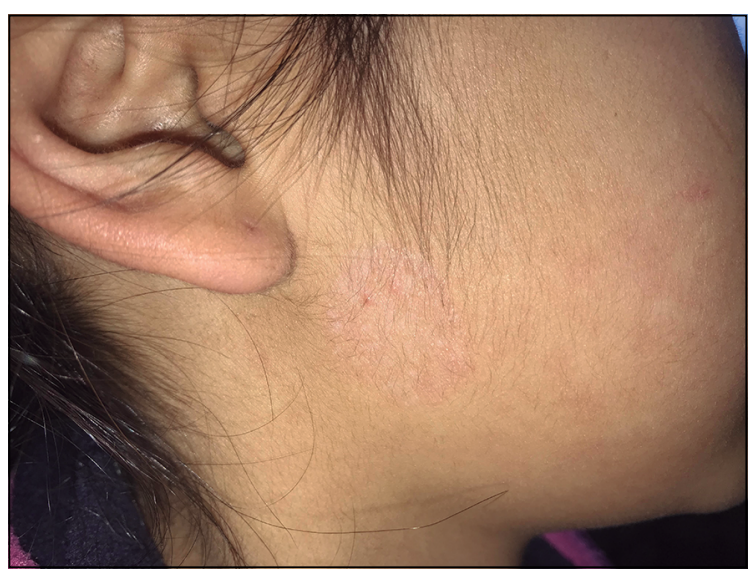

Figura 2. Lesión descamativa, levemente eritematosa y redondeada en el rostro de una niña.

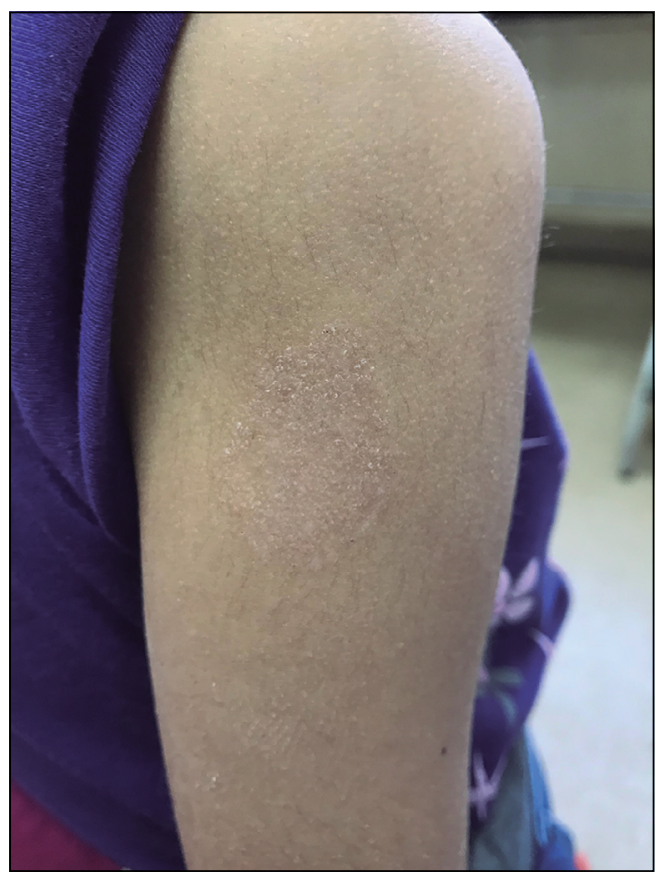

Figura 3. Lesión descamativa, levemente eritematosa y redondeada en el brazo derecho de una niña. 


\section{Resultados}

Hubo 18 casos en total, 16 niños (15 pertenecían al kindergarten y 1 niña de 7 años a otro curso) y dos adultos (madres de los alumnos).

De un total de 22 alumnos que tenía el kindergarten, cinco niños lo abandonaron a raíz del brote. La tasa de ataque en el curso fue de $68 \%$. Nueve casos correspondieron a varones con un promedio de edad de 6 años. Catorce de nacionalidad chilena y 1 haitiana. El caso índice correspondió a un niño chileno de 6 años de edad y el caso primario al niño haitiano de 5 años.

La localización más frecuente de la infección fue en el rostro, seguido por cuero cabelludo (Tabla 1).

De los 18 casos, a 14 (13 niños y un adulto) se les realizó toma de muestra para estudio micológico. En diez casos hubo confirmación micológica, siete con examen micológico directo que mostró presencia de elementos fúngicos y en 10 hubo desarrollo de T. tonsurans. Cuatro pacientes no presentaron examen micológico directo ni cultivo positivo.

Inicialmente, 10 niños y un adulto fueron tratados con clotrimazol crema al 1\%. Sólo en un caso hubo respuesta clínica, requiriendo los demás cambio a terbinafina tópica al $1 \%$ ( 1 casos) o sistémica ( 6 casos), obteniéndose buena respuesta clínica hasta el control del día 30/08/2018. Hubo pérdida de seguimiento de tres de estos niños.

Cinco niños y un adulto recibieron terbinafina desde un principio, ya fuese tópica o sistémica, con buena respuesta clínica.

Sólo un niño no inició tratamiento ni asistió a controles en el centro de salud.

\section{Discusión}

Las dermatofitosis constituyen un conjunto de infecciones frecuentes de la comunidad; cada país tiene especies características, con una prevalencia y formas clínicas de presentación que pueden tener variaciones en el tiempo ${ }^{2,4}$. Esto se ha asociado a cambios en el estilo de vida, migraciones, la aparición de nuevos antifúngicos, tipos de calzado, mejoría de la higiene y la prevención ${ }^{4,11}$. En Chile, en las últimas décadas se ha demostrado cambios, tanto en la frecuencia como en las presentaciones clínicas de las infecciones provocadas por los géneros Microsporum y Epidermophyton ${ }^{11,12}$.

Dentro del género Trichophyton, T. rubrum es el más frecuente en las distintas dermatofitosis reportadas en la mayoría de los países, incluido Chile; no obstante, $T$. tonsurans se considera un agente emergente en varias regiones del mundo ${ }^{6,7,13}$. En América del Sur se ha descrito la circulación de este agente en varios de los países, tanto en dermatofitosis de niños como en adultos ${ }^{7,14-16}$. En
Tabla 1. Distribución de las localizaciones en el total de los infectados con Tricophyton tonsurans

\begin{tabular}{ll}
\hline Localización & $\mathbf{n}$ \\
\hline Rostro & 8 \\
\hline Extremidades superiores & 5 \\
\hline Cuero cabelludo & 4 \\
\hline Tórax & 2 \\
\hline Extremidades inferiores & 2 \\
Generalizado & 1 \\
\hline
\end{tabular}

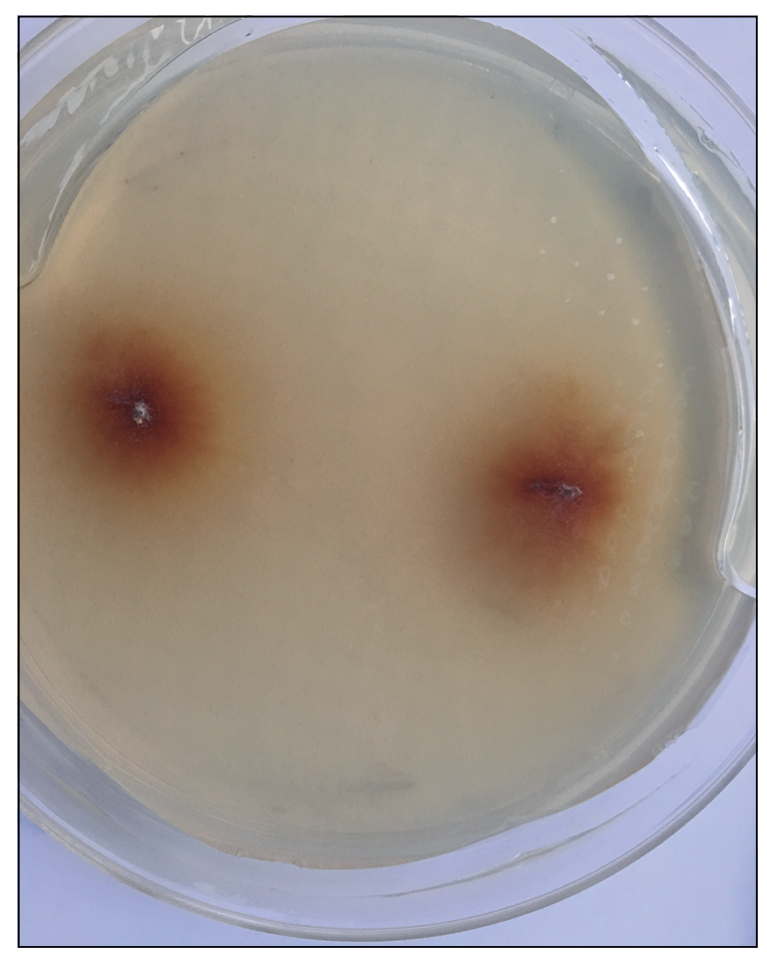

Figura 4. Colonia de aspecto mullida, con pigmento rojizo en el medio (lactrimel) a los 10 días de incubación a $37^{\circ} \mathrm{C}$.

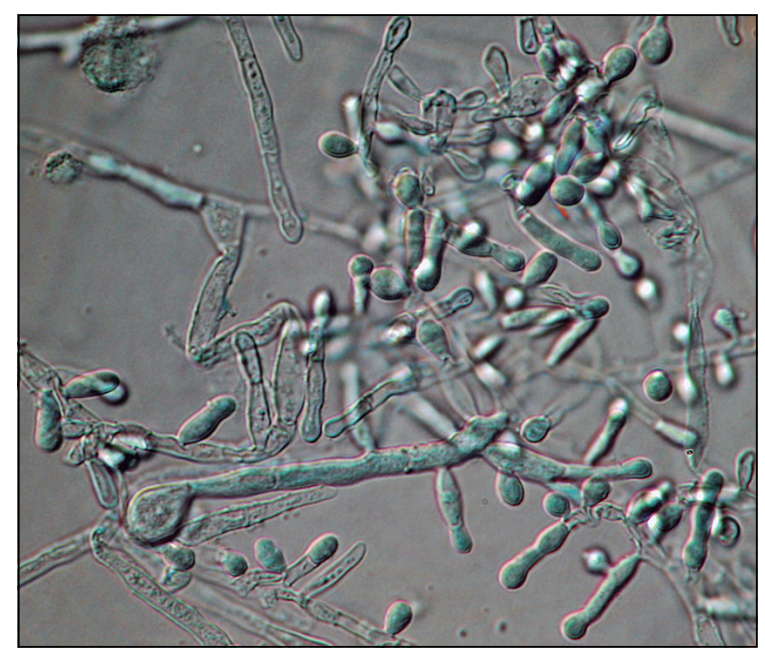

Figura 5. Se observan microconidios sésiles y subglobosos. Clamidospora terminal. Tinción de lactofenol con azul de algodón. 100X. 
Chile se reportó su aparición en el 2012 y se describió un aumento sostenido de los casos hasta el año 2015 en tiña capitis de niños ${ }^{8}$. Nuestro trabajo confirma que este agente sigue circulando en nuestro país y que, además, puede provocar brotes comunitarios de dermatofitosis.

Debido a que $T$. tonsurans es un agente antropofílico y que tiene una mayor transmisibilidad, se han reportado brotes comunitarios y escolares en casi todos los continentes ${ }^{6,17-19}$. Este brote es el primero reportado en nuestro país y refleja lo complejo que pueden llegar a ser este tipo de infecciones, tanto en la trasmisión, diagnóstico y su tratamiento. Además, nos muestra la posible asociación de la circulación de $T$. tonsurans con el aumento de los viajes de los chilenos al extranjero o a la inmigración desde países que presentan con mayor frecuencia este agente como causa de dermatofitosis ${ }^{6,14-16}$.

Se reporta que, en la mayoría de los brotes, la principal localización es en cuero cabelludo ${ }^{17-19}$, situación distinta a lo descrito en este trabajo, ya que la principal localización fue facial, seguida por extremidades superiores y cuero cabelludo. La selectividad con la que las diferentes especies de dermatófitos afectan estructuras queratinizadas se debe a que poseen queratinasas y enzimas proteolíticas específicas para cada una de ellas. Además, se debe considerar que cuando una especie es introducida a una población anteriormente no expuesta a ese agente, puede provocar infecciones con manifestaciones poco frecuentes o conocidas previamente ${ }^{20}$.

El diagnóstico etiológico de la dermatofitosis es fundamental tanto para documentar posibles agentes emergentes, como para guiar el tratamiento antifúngico ${ }^{1-3}$. En nuestro estudio, la mayoría de los niños infectados del curso fue a la toma de muestra el día indicado y el diagnostico se realizó con examen microscópico directo y cultivo.

En las dermatofitosis por T. tonsurans se recomienda el tratamiento con terbinafina debido al menor riesgo de fracaso terapéutico ${ }^{20}$. Si la infección compromete el cuero cabelludo, debe tratarse con terbinafina sistémica por al menos ocho semanas ${ }^{20,21}$. Sólo un paciente de nuestro trabajo tuvo respuesta clínica a clotrimazol crema, los demás debieron tratarse con terbinafina tópica o sistémica, con buena respuesta clínica, lo que coincide con lo recomendado anteriormente.

Debido a los constantes cambios demográficos, migraciones y modificaciones en la calidad de vida de las poblaciones, se hace necesario determinar probables cambios en la frecuencia de los dermatofitos y otras especies de importancia clínica que pueden ingresar y afectar a la población en general.

\section{Conclusiones}

Se reporta el primer brote comunitario de tiña por T. tonsurans en Chile. Hubo 18 casos, 16 niños y dos adultos. Nueve casos fueron varones, con promedio de edad de 6 años, la mayoría de nacionalidad chilena. La localización más frecuente de la infección fue en el rostro y la mayoría de los casos requirió tratamiento con terbinafina por fracaso terapéutico con clotrimazol. Trichophyton tonsurans es un agente emergente en nuestro país y que provoca brotes de dermatofitosis en distintos países del mundo.

\section{Resumen}

Introducción: Las tiñas son infecciones que afectan la piel y anexos. Trichophyton tonsurans es un hongo antropofílico emergente que puede provocar brotes. Objetivo: Describir un brote de tiña por T. tonsurans en una escuela de Valparaíso. Materiales y Método: Estudio descriptivo realizado entre junio-agosto de 2018. Se consideró caso a todo alumno de la escuela y a familiares directos en contacto con ellos, con diagnóstico clínico de tiña. El estudio micológico de las muestras tomadas se realizó a través de pruebas morfo-fisiológicas. Los datos se expresaron en números y porcentajes. Resultados: Hubo 18 casos, 16 niños (15 del kindergarten) y dos adultos. Tasa de ataque en kindergarten fue de $68 \%$. Nueve casos fueron varones con promedio de edad de 6 años. Catorce de nacionalidad chilena y una haitiana. El caso índice fue un niño chileno y el primario el niño haitiano. La localización más frecuente fue en el rostro. Diez casos tuvieron cultivo con desarrollo de $T$. tonsurans. La mayoría requirió tratamiento con terbinafina por fracaso terapéutico con clotrimazol. Conclusiones: El brote por T. tonsurans ocurrió principalmente en varones del kindergarten. La principal localización fue en el rostro. La mayoría de los casos requirió terbinafina como tratamiento por fracaso terapéutico con clotrimazol.

\section{Referencias bibliográficas}

1.- Velasco M, García-Melgares L, Gimeno E, Roche E, Vilata J J. Dermatofitosis. Vilata J J, editor. Micosis Cutaneas, 1a ed. Madrid: Editorial Médica Panamericana 2006; p. 49-72.

2.- Molina de Diego A. Aspectos clínicos, diagnósticos y terapéuticos de las dermatofitosis. Enferm Infecc Microbiol Clin 2011; 29 (3): 33-9. https://doi.org/10.1016/ S0213-005X(11)70025-8.

3.- de Hoog G S, Dukik K, Monod M, Packeu A, Stubbe D, Hendrickx M, et al. Toward a novel multilocus phylogenetic taxonomy for the
Dermatophytes. Mycopathologia. 2017; 182 (1-2): 5-31 https://doi.org/10.1007/s11046-0160073-9.

4.- Cruz R, Ponce E, Calderón L, Delgado N, Vieille P, Piontelli E. Micosis superficiales en la ciudad de Valparaíso, Chile: Período 2007-2009. Rev Chilena Infectol 2011; 28 
(5): 404-9. https://dx.doi.org/10.4067/S071610182011000600002.

5.- Piontelli E, Toro M, Casanova D, Jara D. Micosis superficiales en pacientes de servicios dermatológicos de la V Región: Estudios de prevalencia en el período 1984-1989. Bol Micol 1991; 6: 63-8. https://micologia.uv.cl/index. $\mathrm{php} /$ Bolmicol/article/view/1613/1670.

6.- Raccurt C P, Dorsainvil D, Boncy M, Boncy J, Auguste G. The emergence of Trichophyton tonsurans in Port-au-Prince, Haiti. Med Mycol 2009; 47 (2): 197-200. https://doi. org/10.1080/13693780802192676.

7.- van Gelderen A. Tinea capitis inflamatoria por Trichophyton tonsurans var. Sulfureum. Bol Micol 2012; 27 (2): 39-45. https://revistas.uv.cl/ index.php/Bolmicol/article/view/887/864.

8.- Aranibar L, Stevens J, Palma C, Zapata S, Pizarro J. Estudio epidemiológico de la tinea capitis en una población de Santiago de Chile. La aparición de Trichophyton tonsurans como agente etiológico. Piel 2017; 32 (10): 604-9. https://doi.org/10.1016/j. piel.2017.06.007.

9.- de Hoog G S, Guarro J, Gene J, Figueras M J. Atlas of Clinical Fungi version 4.1.4. http:// atlas.clinicalfungi.org/AtlasOnline (Acceso el 1 de octubre de 2018).

10.- MycoBank Database. http://www.mycobank. org (Acceso el 1 de octubre de 2018).

11.- Cruz R, Carvajal L, Pérez S, Rodríguez V. Aislamiento de Microsporum spp. en dermatofitosis en pacientes de la Región de Valparaíso. Chile. Rev Argent Dermatol. 2017; 98 (1): 27-37. https://rad-online.org. ar/2017/04/01/aislamiento-de-microsporumspp-en-dermatofitosis-en-pacientes-de-laregion-de-valparaiso-chile/

12.- Cruz R, Carvajal L. Frecuencia de Epidermophyton floccosum en dermatofitos aislados en un laboratorio de la Región de Valparaíso, Chile. Período 1980-2010. Rev Chil Infectol. 2018; 35 (3): 262-5. http://dx.doi. org/10.4067/s0716-10182018000300262.

13.- Lee W J, Sim H B, Jang Y H, Lee S J, Kim D W, Jun J B, et al. Skin infection due to Trichophyton tonsurans still occurs in people in Korea but not as outbreaks. JKMS 2016; 31(2), 296-300. https://doi.org/10.3346/ jkms.2016.31.2.296.

14.- Benavides J, Villanueva J. Tiña del cuero cabelludo por Trichophyton tonsurans: agente emergente en Colombia. Rev Asoc Colomb Dermatol 2010; 18: 99-101. https:// revistasocolderma.org/sites/default/files/tina del_cuero_cabelludo.pdf.

15.- Mata S, Carrillo Y, Canache O, Larrea F, Collela M T, Pineda V, et al. Epidemiología de tinea capitis en Venezuela. Dermatol Venez. 2013; 51: 15-9. http://revista.svderma.org/ index.php/ojs/article/view/514.

16.- Sidrim J J, Rocha M F, Leite J J, Maranhão F C, Lima R A, Castelo-Branco D de S, et al. Trichophyton tonsurans strains from
Brazil: phenotypic heterogeneity, genetic homology, and detection of virulence genes. Can J Microbiol. 2013; 59: 754-60. https://doi. org/10.1139/cjm-2013-0670.

17.- Shroba J, Abdel-Rahman S M. A large outbreak of Trichophyton tonsurans among healthcare workers in a pediatric hospital. Am J Infect Control 2009; 37(1): 43-8. https://doi. org/10.1016/j.ajic.2007.11.008.

18.- Moreno M, Valdez P, García A, Arenas R. Brote epidémico intrafamiliar de tiña de la cabeza por Trichophyton tonsurans: informe de cuatro casos en tres generaciones. Med Cutan Iber Lat Am 2015; 43 (3): 217-21. http:// www.medigraphic.com/cgi-bin/new/resumen. cgi?IDARTICULO $=65262$.

19.- Ruiz S, Guijarro E, Cardona A, Hernández M A, Muniain M P, Martín A $\mathrm{M}$, et al. Epidemia de tiña por Trichophyton tonsurans en una escuela. Rev Pediatr Aten Primaria. 2016;18: 325-31. http:// scielo.isciii.es/pdf/pap/v18n72/1139-7632pap-18-72-00325.pdf.

20.- Padilla M, Alfaro L, Cardona M. Tiña por Trichophyton tonsurans en diversas topografías. Revisión de la literatura. Rev Cent Dermatol Pascua. 2011; 20: 46-53. http://www. medigraphic.com/pdfs/derma/cd-2011/cd112c. pdf.

21.- Kelly B P. Superficial fungal infections. Pediatr Rev 2012; 33(4):22-37. http://doi.org/ 10.1542/ pir.33-4-e22. 